\title{
Editorial: The inaugural issue of Socio-Environmental Systems Modelling (SESMO)
}

\author{
Anthony J. Jakeman ${ }^{1 *}$, loannis Athanasiadis², Marjolijn Haasnoot ${ }^{3}$, Marco Janssen ${ }^{4}$, Alexey Voinov ${ }^{5}$ \\ ${ }^{1}$ Australian National University, Australia \\ ${ }^{2}$ Wageningen University \& Research, The Netherlands \\ ${ }^{3}$ Deltares, Delft University of Technology, The Netherlands \\ ${ }^{4}$ Arizona State University, USA \\ ${ }^{5}$ University of Technology Sydney, Australia
}

\section{Making progress in resolving grand challenge environmental issues}

The world's major environmental issues are wicked ones where: decision making and management is fraught with pervasive and deep uncertainties; stakes are contested between interest groups, government levels and industrial sectors; and cumulative scales and the dynamic nature of environmental and socioeconomic impacts need to be considered in assessing tradeoffs for supporting decisions. These issues are often framed as "socioenvironmental" in nature and as grand challenges, due to the scale and severity of the issues, their complexity and uncertainty of interactions between humans and the environment.

Grand challenge issues are typically influenced by combinations of population growth, globalization, urbanization, technological developments, institutional capacity, and climate change and variability. Such issues include, for example:

- Addressing conflicts between water, energy, land and food security (in urban and rural contexts)

- Efficient and effective use of water, land and energy to ensure food security, resilience of ecosystems, and sustainable livelihoods

- Balancing mitigation and adaptation opportunities to avoid the adverse consequences of global change, and their interactions with Sustainable Development Goals

- Protection of air quality in over-polluted cities

- Resilience of marine and freshwater aquatic systems

- Preservation of ecosystem services from land and water

To make progress in resolving such grand challenge issues, Integrated Assessment and model-based processes are essential as a scientific "meta-discipline" or "transdiscipline" that integrates disciplinary and sectoral knowledge about the relevant problem domain and makes it available for societal learning and decision making processes. In all socio-environmental investigations, conceptual and numerical modelling are essential to systemically integrate knowledge and opportunities - from all influencing sectors, interest groups and stakeholders in general. Modelling is also required to help assimilate and share knowledge, generate trust and improve adoption prospects. Socio-Environmental Modelling (SESMO) seeks to showcase innovations in modelbased research toward resolving these grand challenge problems, thereby filling the niche of this rapidly evolving field of science.

Correspondence:
Contact A. Jakeman at tony.jakeman@anu.edu.au

Cite this article as:

Jakeman, A.J., Athanasiadis, I., Haasnoot, M., Janssen, M. \& Voinov, A.

Editorial: The inaugural issue of Socio-Environmental Systems Modelling (SESMO)

Socio-Environmental Systems Modelling, vol. 1, 2019, doi:10.18174/sesmo.16399

This work is licensed under a Creative Commons Attribution-NonCommercial 4.0 International License. 


\section{Journal aims and scope}

SESMO aims to progress our understanding, learning and decision making on major socio-environmental issues using advances in fit-for-purpose modelling and model-based processes that engage with institutional and governance contexts, and include cross-sectoral and scale challenges, and stakeholder perspectives.

The journal intends to advance model-based processes and their wider application to a new level that leads to innovations in thinking and practice to support resolution of grand challenge problems, including generating policy insights and evidence, and reducing and managing critical uncertainties. To this end, problem framing, model development and evaluation at various time and spatial scales as well as eclectic uncertainty analysis are stressed so that the advantages and limitations of model-related assumptions are transparent. SESMO also promotes knowledge and information sharing, not only through open access to all journal articles, but also by encouraging authors to make their code and data publicly available.

SESMO seeks papers that address how science and research-oriented modellers can help identify and provide germane information and support required by managers and decision-makers, including those papers presenting:

1. Frameworks, methodologies, and/or case studies that help us discover evidence for workable solutions at various spatial scales (local to global) and time scales (operational to future management), recognising that these will emerge from social and other science perspectives.

2. Methods that help us understand which uncertainties are important and to track uncertainties that arise from things like problem framing, modelling, and model evaluation; especially methods that simultaneously and separately consider identifying and managing uncertainty from the many sources and facets of the problem.

3. Analysis of existing decision-support models - such as integrated assessment models or domain models, say, in climate change, water and air quality - to assess weaknesses and guide improvements, and establish gains and losses from adding increased complexity to established models.

4. Insights into how users and decision makers can be engaged to influence technical efforts.

5. Quantitative and qualitative methodologies and ontologies for all parts of the modelling process from problem framing and conceptual modelling to evaluation of options and communicating tradeoffs among potential solutions that allow for feedback between scientists, decision-makers and the wider public. These may include decision-support systems, model coupling and integration techniques, web interfaces, big data analysis, optimization, stakeholder engagement methods, visioning, narrative, and visualization techniques.

6. Methods for soliciting information from stakeholders, individuals, groups, agents, and communication and facilitation tools to feed the information back into stakeholder discussions and processes, including innovative approaches for developing and handling surveys, interviews and mental model mapping techniques.

\section{Article categories}

\section{Research papers}

Articles in this category present the results of original research in the area of socio-environmental systems. The core article should desirably not exceed 10,000 words but could include appendices with additional technical details and analysis. All manuscripts will be peer-reviewed and need to meet the data and code availability standards upheld by SESMO.

\section{Advances in Modelling Practice}

Articles in this category are short, succinct, and problem-oriented offering tangible take-aways that can be applied to the day-to-day work of modellers of socio-environmental systems. Topics can include, but are not limited to: innovations and best practices in modelling software, participatory modelling, programming solutions, communicating model results, model analysis, model evaluation, and model documentation. We envision two types of articles. First, the traditional research articles which define a problem or issue in the practice of modelling socio-environmental systems, and then demonstrate how that problem was overcome. 
The second type of article is a "how-to" article where the intent is to provide the reader with clear, concise, step-by-step guidelines for successfully completing tasks that are or might become common practice. All manuscripts will follow the traditional peer-review process and need to meet the journal's data and code availability standards.

\section{Modelling reviews}

Articles in this category are reviews of books, models, data, methods, conferences, MOOCs or other types of scholarly activities related to modelling of socio-environmental systems. Reviews provide authoritative, unbiased critique of those scholarly activities and provide constructive information of the latest developments in the field. 